\title{
ABSOLUTE CONTINUITY OF THE SPECTRUM FOR PERIODICALLY MODULATED LEAKY WIRES IN $\mathbb{R}^{3}$
}

\author{
PAVEL EXNER AND RUPERT L. FRANK
}

\begin{abstract}
We consider a model of leaky quantum wire in three dimensions. The Hamiltonian is a singular perturbation of the Laplacian supported by a line with the coupling which is bounded and periodically modulated along the line. We demonstrate that such a system has a purely absolutely continuous spectrum and its negative part has band structure with an at most finite number of gaps. This result is extended also to the situation when there is an infinite number of the lines supporting the perturbations arranged periodically in one direction.
\end{abstract}

\section{INTRODUCTION}

Existence of transport in quantum systems having a periodic structure is important in many areas, particularly in condensed-matter physics. Mathematically this property is expressed as the absolute continuity of spectrum of the appropriate Hamiltonian. For Schrödinger operators with regular potentials which are "completely" periodic in the sense that the basic period cell is compact the problem is well understood - cf. [T] or [RS], Sec.XIII.16.

Recently a class of models attracted attention in which one or both of the above conditions are violated. They concern thin microscopic semiconductor structures, often dubbed "quantum wires", which are intensively studied as construction elements of future electronic devices. Comparing to the usual treatment of such objects the mentioned models are realistic in the sense that they describe the wires by elongated "potential wells" so that quantum tunneling is not suppressed. On the other hand, they are often idealized using singular potentials with the aim to make the model solvable; the corresponding Hamiltonian can be written formally as

$$
-\Delta+\sigma(x) \delta(x-\Gamma)
$$

in $L_{2}\left(\mathbb{R}^{\nu}\right), \nu=2,3$, where $\Gamma$, typically a curve or a family of curves, supports the interaction. Various spectral properties of such operators have been derived in several last years, see [AGHH] for a bibliography.

On the other hand, many questions are still open. For instance, while it is natural to conjecture that the spectrum is absolutely continuous when $\Gamma$ is a periodic curve and $\sigma$ is constant along it, only a partial result is known $\mathrm{BeDuE}$ and a full proof is missing. The situation is better in the case when the periodicity concerns the coupling rather than the geometry of the

Date: February 2, 2008. 
interaction support. If $\Gamma$ is a straight line in $\mathbb{R}^{2}$ and $\sigma$ is periodic the sought property can be obtained by modification of the results of [Fr1, Fr2, FrSh] (recall also that a similar result for Schrödinger operators with a "partially periodic" regular potential was derived in [FiKl]). It is important, however, that the codimension of $\Gamma$ is one here and the Hamiltonian can be defined naturally through the associated quadratic form.

The main aim of this paper is to solve the analogous problem for a straight line in $\mathbb{R}^{3}$. In this case the codimension is two and the operator has to be defined by means of boundary conditions involving generalized boundary values as in EKo1. We will be able to demonstrate that such a Hamiltonian has a purely absolutely continuous spectrum, and moreover, that its negative part has band structure with an at most finite number of gaps. The corresponding generalized eigenfunctions are of physical interest, of course, because they describe states guided along the "wire". Moreover, we are going to extend the result to the case where the interaction support consists of an infinite family of parallel and equidistant straight lines in a fixed plane ${ }^{1}$.

The paper is organized as follows. The main results are formulated in the next section and proved subsequently in Secs. 3. 5. The results concerning the extended model are stated in Sec. 6] and proved in the rest of the paper.

\section{MAin RESUlts}

2.1. Description of the results. In $\mathbb{R}^{3}$ we introduce coordinates $(x, y)$, $x \in \mathbb{R}, y \in \mathbb{R}^{2}$, and denote $\Gamma:=\mathbb{R} \times\{(0,0)\}$. Moreover, let $\sigma$ be a realvalued, $2 \pi$-periodic function such that

$$
\sigma \in L_{\infty}(\mathbb{R}) .
$$

We will construct a self-adjoint operator $H$ in $L_{2}\left(\mathbb{R}^{3}\right)$ corresponding to the formal expression (1.1) which can be written ${ }^{2}$ also as $-\Delta+\sigma(x) \delta(|y|)$. Put $C_{\epsilon}:=\left\{(x, y) \in \mathbb{R}^{3}:|y| \leq \epsilon\right\}$ for $\epsilon>0$. We consider functions $u \in L_{2}\left(\mathbb{R}^{3}\right)$ such that

$$
u \in H^{2}\left(\mathbb{R}^{3} \backslash C_{\epsilon}\right) \quad \text { for all } \epsilon>0 .
$$

By the embedding theorems $u$ is continuous in $\mathbb{R}^{3} \backslash \Gamma$ and hence it restriction $u(., y)$ to the line $\{(x, y): x \in \mathbb{R}\}$ is well-defined. We denote by $\Upsilon$ the class of functions $u \in L_{2}\left(\mathbb{R}^{3}\right)$ satisfying (2.2) and such that the limits

$$
\Xi u:=-\lim _{y \rightarrow 0} \frac{1}{\log |y|} u(., y), \quad \Omega u:=\lim _{y \rightarrow 0}(u(., y)+\log |y| \Xi u),
$$

\footnotetext{
${ }^{1}$ We leave out in this paper another possible extension to the situation when the line family is periodic in two different directions. In this case the basic period cell is compact and more conventional methods can be used.

${ }^{2}$ It has to be stressed that this expression is formal and the proper way to introduce $\sigma$ is given by (2.6) below; recall that the absence of the coupling means $\sigma=\infty$.
} 
exist in the sense of distributions and belong to $L_{2}(\mathbb{R})$.

We will recall in Subsection 3.1 how one constructs a self-adjoint operator $H$ in $L_{2}\left(\mathbb{R}^{3}\right)$ such that

$$
\begin{aligned}
H u & =-\Delta u \quad \text { in } \mathbb{R}^{3} \backslash \Gamma, \\
\mathcal{D}(H) & =\left\{u \in \Upsilon: \Delta u \in L_{2}\left(\mathbb{R}^{3} \backslash \Gamma\right), \Omega u-2 \pi \sigma \Xi u=0\right\} .
\end{aligned}
$$

(Recall that $\Delta u \in L_{2}\left(\mathbb{R}^{3} \backslash \Gamma\right)$ means that the distribution $\Delta u$ is a function on $\mathbb{R}^{3} \backslash \Gamma$ and square-integrable. We do not make an assertion about its nature on $\Gamma$.)

Our main result is

Theorem 2.1. The spectrum of the operator $H$ is purely absolutely continuous.

For the proof of this theorem we will investigate the scattering between $H$ and $H_{0}$, the standard self-adjoint realization of $-\Delta$ in $\mathbb{R}^{3}$. Recall the definition (in case of existence) of the wave operators (see, e.g., Ya])

$$
W_{ \pm}:=s-\lim _{t \rightarrow \pm \infty} \exp (i t H) \exp \left(-i t H_{0}\right) .
$$

We will prove

Theorem 2.2. The wave operators $W_{+}$and $W_{-}$exist, satisfy $\mathcal{R}\left(W_{+}\right)=$ $\mathcal{R}\left(W_{-}\right)$and are not complete.

The existence of the wave operators implies, as it is well-known, that $\sigma_{a c}\left(H_{0}\right)=[0, \infty)$ is contained in the absolutely continuous spectrum of the operator $H$. Moreover, we note that the identity $\mathcal{R}\left(W_{+}\right)=\mathcal{R}\left(W_{-}\right)$implies the unitarity of the scattering matrix. The non-completeness of the wave operators is due to guided states, i.e. states that are localized near the wire $\Gamma$ for all times. They correspond to bands in the (negative) spectrum of $H$. We will prove

Theorem 2.3. The negative spectrum of the operator $H$ is non-empty and has band structure with at most finitely many gaps.

However, we emphasize that guided states correspond not only to negative energies. Indeed, if $\sigma \equiv \alpha \in \mathbb{R}$ is constant then the spectrum of $H$ on $\mathcal{R}\left(W_{ \pm}\right)^{\perp}$ coincides with the half-line $[\xi(\alpha), \infty)$ where

$$
\xi(\alpha)=-4 e^{2(-2 \pi \alpha+\psi(1))}, \quad \alpha \in \mathbb{R},
$$

and $-\psi(1)$ is the Euler constant (numerically, $-\psi(1)=0.577 \ldots$ ). It is a natural question whether the spectrum of $H$ on $\mathcal{R}\left(W_{+}\right)^{\perp}$ is bounded when $\sigma$ is non-constant ${ }^{3}$.

Remark 2.4. The subspace $\mathfrak{M}$ of functions being rotationally symmetric with respect to the variable $y$ reduces both $H$ and $H_{0}$, and the parts of these operators in $\mathfrak{M}^{\perp}$ coincide. In particular, guided states belong to $\mathfrak{M}$.

\footnotetext{
${ }^{3}$ In the case of a constant $\sigma$ the positive-energy guided states are expected be unstable with respect to perturbations, but we are not going to discuss this problem here.
} 
Remark 2.5. Of course, the assumption that the period is $2 \pi$ is not essential. Moreover, the assumption (2.1) can be relaxed to assuming that for every $\epsilon>0$ there is a $C_{\epsilon}>0$ such that

$$
\|\sigma f\|_{L_{2}(-\pi, \pi)}^{2} \leq \epsilon \sum_{n \in \mathbb{Z}}\left(\log \left(1+n^{2}\right)\right)^{2}\left|\hat{f}_{n}\right|^{2}+C_{\epsilon}\|f\|_{L_{2}(-\pi, \pi)}^{2}
$$

for all smooth, $2 \pi$-periodic functions $f$ with Fourier coefficients $\hat{f}_{n}$, see (3.3) below.

2.2. Direct integral decomposition. Because of periodicity the operator $H$ can be partially diagonalized. A fundamental cell is the layer

$$
\Pi:=\left\{(x, y) \in \mathbb{R}^{3}: x \in[-\pi, \pi)\right\} .
$$

Actually, we will only work with functions on $\Pi$ that are periodic with respect to the variable $x$ and one may think of $\Pi$ as a manifold with opposite points on the planes $\{x=\pi\}$ and $\{x=-\pi\}$ identified, but we ignore this for the sake of simplicity. However, we will identify $\Pi \cap \Gamma=[-\pi, \pi) \times\{(0,0)\}$ with the 'torus' $\mathbb{T}$.

By $\tilde{H}^{2}(\Pi)$ we denote the class of functions $u \in H^{2}(\Pi)$ the periodic extension of which belongs to $H_{l o c}^{2}\left(\mathbb{R}^{3}\right)$, and by $\tilde{\Upsilon}$ we denote the class of functions $u \in L_{2}(\Pi)$ satisfying

$$
u \in H^{2}\left(\Pi \backslash C_{\epsilon}\right) \quad \text { for all } \epsilon>0
$$

and such that their periodic extension belongs to $\Upsilon_{l o c}$. Here as usual if $F$ is a class of functions on $\mathbb{R}^{3}$ then $F_{\text {loc }}:=\left\{u: \mathbb{R}^{3} \rightarrow \mathbb{C}: \varphi u \in F \forall \varphi \in C_{0}^{\infty}\left(\mathbb{R}^{3}\right)\right\}$. For $u \in \tilde{\Upsilon}$ the functions $\Xi u, \Omega u$ are well-defined and belong to $L_{2}(\mathbb{T})$. We will recall in Subsection 3.2 that there exists a family of self-adjoint operators $H(k), k \in Q:=\left[-\frac{1}{2}, \frac{1}{2}\right)$, in $L_{2}(\Pi)$ such that

$$
\begin{aligned}
H(k) u & =\left(\left(D_{x}+k\right)^{2}+D_{y}^{2}\right) u \quad \text { in } \Pi \backslash \Gamma, \\
\mathcal{D}(H(k)) & =\left\{u \in \tilde{\Upsilon}: \Delta u \in L_{2}(\Pi \backslash \Gamma), \Omega u-2 \pi \sigma \Xi u=0\right\} .
\end{aligned}
$$

Here $D_{x}=-i \frac{\partial}{\partial x}, D_{y}=-i \nabla_{y}$. Moreover, we denote by $H_{0}(k)$ the operator $\left(D_{x}+k\right)^{2}+D_{y}^{2}$ in $L_{2}(\Pi)$ with domain $\tilde{H}^{2}(\Pi)$.

The Gelfand transformation is initially defined for $u \in C_{0}^{\infty}\left(\mathbb{R}^{3}\right)$ by

$$
(\mathcal{U} u)(k, x, y):=\sum_{n \in \mathbb{Z}} e^{-i k(x+2 \pi n)} u(x+2 \pi n, y), \quad k \in Q,(x, y) \in \Pi,
$$

and extended by continuity to a unitary operator $\mathcal{U}: L_{2}\left(\mathbb{R}^{3}\right) \rightarrow \int_{Q}^{\oplus} L_{2}(\Pi) d k$. It is well-known that

$$
\mathcal{U} H_{0} \mathcal{U}^{*}=\int_{Q}^{\oplus} H_{0}(k) d k
$$

In Subsection 3.2 we will prove that similarly

$$
\mathcal{U} H \mathcal{U}^{*}=\int_{Q}^{\oplus} H(k) d k .
$$


This reduces the investigation of the operator $H$ to the study of the fiber operators $H(k)$.

2.3. Results about the fiber operators. Information about the continuous spectrum of the operators $H(k)$ can be obtained by scattering theory for the pair $\left(H(k), H_{0}(k)\right)$. Note that the operator $H_{0}(k)$ can be diagonalized explicitly. Its spectrum is purely absolutely continuous and coincides with $\left[k^{2}, \infty\right)$. The spectral multiplicity is finite and changes at the points from the threshold set

$$
\tau(k):=\left\{(n+k)^{2}: n \in \mathbb{Z}\right\} .
$$

We introduce the wave operators

$$
W_{ \pm}(k):=s-\lim _{t \rightarrow \pm \infty} \exp (i t H(k)) \exp \left(-i t H_{0}(k)\right) .
$$

Proposition 2.6. Let $k \in Q$. Then the wave operators $W_{+}(k)$ and $W_{-}(k)$ exist and are complete. In particular, $\sigma_{a c}(H(k))=\left[k^{2}, \infty\right)$.

By deriving a limiting absorption principle we will show

Proposition 2.7. Let $k \in Q$. Then $\sigma_{s c}(H(k))=\emptyset$.

Concerning the point spectrum of the fiber operators we prove

Proposition 2.8. Let $k \in Q$. Then $\sigma_{\text {disc }}(H(k))=\sigma(H(k)) \cap\left(-\infty, k^{2}\right)$ is non-empty and finite.

Indeed, we will prove that $H(k)$ has an eigenvalue less or equal $\xi(\tilde{\sigma})+k^{2}$ where $\xi$ is given by (2.5) and

$$
\tilde{\sigma}:=\frac{1}{2 \pi} \int_{\mathbb{T}} \sigma(x) d x .
$$

Moreover, for the proof of absolute continuity we need

Proposition 2.9. There exists a countable family of open connected sets $U_{j}, V_{j} \subset \mathbb{R}$ and real-analytic functions $h_{j}: U_{j} \times V_{j} \rightarrow \mathbb{C}$ satisfying

(1) for all $j$ and all $\lambda \in U_{j}$ one has $h_{j}(\lambda,.) \not \equiv 0$, and

(2) $\left\{(\lambda, k) \in \mathbb{R} \times Q: \lambda \in \sigma_{p}(H(k))\right\} \subset \bigcup_{j}\left\{(\lambda, k) \in U_{j} \times V_{j}: h_{j}(\lambda, k)=\right.$ $0\}$.

2.4. Reduction to the fiber operators. Our main results Theorems 2.1 -2.3 can be deduced from Propositions 2.6-2.9 in a standard way. We only sketch the major steps.

Proof of Theorem 2.1. Propositions 2.6, 2.7 and 2.9 allow us to follow the proof of Theorem 1.4 in [Fr2] word by word.

Proof of Theorem 2.3. We will see below that the discrete eigenvalues of $H(k)$ depend piecewise analytically on $k$. Hence the existence of negative spectrum of $H$ and its band structure follow from Proposition 2.8 and the decomposition (2.8). By analytic perturbation theory there can be at most one gap in $\sigma(H)$ between two consecutive eigenvalues of $H(0)$. Hence the finiteness of gaps follows again from Proposition 2.8. 
Proof of Theorem [2.2. Proposition 2.6 implies the existence of the wave operators $W_{ \pm}$and the equality of their ranges (see [Fr1]). The non-completeness follows immediately from Theorems 2.1 and 2.3

\section{Definition of the operators}

3.1. Definition of the operator $H$. Recall that the domain of $H_{0}$ is $H^{2}\left(\mathbb{R}^{3}\right)$ and that the trace operator $\gamma: H^{2}\left(\mathbb{R}^{3}\right) \rightarrow L_{2}(\mathbb{R})$,

$$
\gamma u:=\left.u\right|_{\Gamma},
$$

is well-defined. (Here we identify $\Gamma$ naturally with $\mathbb{R}$.)

For $z \in \mathbb{C} \backslash[0, \infty)$ we consider the pseudo-differential operator $T(z)$ in $L_{2}(\mathbb{R})$,

$$
\begin{aligned}
T(z) & :=\frac{1}{4 \pi} \log \left(D^{2}-z\right)-\varsigma I, \\
\mathcal{D}(T(z)) & :=\left\{f \in L_{2}(\mathbb{R}): \int_{\mathbb{R}}\left(\log \left(1+\xi^{2}\right)\right)^{2}|\hat{f}(\xi)|^{2} d \xi<\infty\right\},
\end{aligned}
$$

where $\varsigma=\frac{1}{2 \pi}(\ln 2+\psi(1))$ and $-\psi(1)$ is as before the Euler constant. Here and in all the following we choose the principal branch of the logarithm on $\mathbb{C} \backslash(-\infty, 0]$. (Note that we have changed the sign in the definition of $T(z)$ as compared to EKo1.)

We write $R_{0}(z):=\left(H_{0}-z I\right)^{-1}$. One checks easily that for $z, \zeta \in \mathbb{C} \backslash[0, \infty)$

$$
T(\bar{z})=T(z)^{*}, \quad T(z)-T(\zeta)=(\zeta-z)\left(\gamma R_{0}(\zeta)\right)\left(\gamma R_{0}(\bar{z})\right)^{*} .
$$

By abstract arguments of $\underline{\mathrm{Po}}$ (see also EKo1) this implies that there exists a self-adjoint operator $H$ in $L_{2}\left(\mathbb{R}^{3}\right)$ such that

$$
\{z \in \mathbb{C} \backslash[0, \infty): 0 \in \rho(T(z)+\sigma)\} \subset \rho(H)
$$

and such that the resolvent $R(z):=(H-z I)^{-1}$ is related to $R_{0}(z)$ by

$$
\begin{aligned}
R(z)= & R_{0}(z)+\left(\gamma R_{0}(\bar{z})\right)^{*}(T(z)+\sigma)^{-1} \gamma R_{0}(z), \\
& z \in \mathbb{C} \backslash[0, \infty), 0 \in \rho(T(z)+\sigma) .
\end{aligned}
$$

By (2.1) the operator $T(-a)+\sigma$ is positive definite for all sufficiently large $a$, and hence $H$ is lower semibounded. Moreover, it was shown in EKo1] that the operator defined in this way satisfies (2.3). Without reproducing the proof here we note that it relies on the identities

$$
\Xi\left(\gamma R_{0}(\bar{z})\right)^{*}=\frac{1}{2 \pi} I, \quad \Omega\left(\gamma R_{0}(\bar{z})\right)^{*}=-T(z) .
$$

3.2. Definition of the operators $H(k)$. Recall that functions in the domain $\tilde{H}^{2}(\Pi)$ of $H_{0}(k)$ satisfy periodic boundary conditions with respect to the variable $x$, and that we identify $\Gamma \cap \Pi$ with $\mathbb{T}$. We use the same notation $\gamma$ for the trace operator $\tilde{H}^{2}(\Pi) \rightarrow L_{2}(\mathbb{T})$. 
Fix $k \in Q$. For $z \in \mathbb{C} \backslash\left[k^{2}, \infty\right)$ we consider the pseudo-differential operator $T(z, k)$ in $L_{2}(\mathbb{T})$,

$$
\begin{aligned}
T(z, k) & :=\frac{1}{4 \pi} \log \left((D+k)^{2}-z\right)-\varsigma I, \\
\mathcal{D}(T(z, k)) & :=\left\{f \in L_{2}(\mathbb{T}): \sum_{n \in \mathbb{Z}}\left(\log \left(1+n^{2}\right)\right)^{2}\left|\hat{f}_{n}\right|^{2}<\infty\right\} .
\end{aligned}
$$

Here

$$
\hat{f}_{n}:=\frac{1}{\sqrt{2 \pi}} \int_{\mathbb{T}} f(x) e^{-i n x} d x, \quad n \in \mathbb{Z},
$$

denote the Fourier coefficients of $f \in L_{2}(\mathbb{T})$, in terms of which the action of $T(z, k)$ is given by

$$
(T(z, k) f)_{n}^{-}=\left(\frac{1}{4 \pi} \log \left((n+k)^{2}-z\right)-\varsigma\right) \hat{f}_{n}, \quad n \in \mathbb{Z} .
$$

Our next goal is to construct the operators $H(k)$ and to verify the direct integral decomposition (2.8). For this we introduce the unitary operator $\tilde{\mathcal{U}}: L_{2}(\mathbb{R}) \rightarrow \int_{Q}^{\oplus} L_{2}(\mathbb{T}) d k$, defined for $f \in C_{0}^{\infty}(\mathbb{R})$ by

$$
(\tilde{\mathcal{U}} f)(k, x):=\sum_{n \in \mathbb{Z}} e^{-i k(x+2 \pi n)} f(x+2 \pi n), \quad k \in Q, x \in \mathbb{T} .
$$

We note that on $H^{2}\left(\mathbb{R}^{3}\right)$ one has the identity

$$
\gamma \mathcal{U}=\tilde{\mathcal{U}} \gamma
$$

where $\gamma$ is the trace operator $\int_{Q}^{\oplus} \tilde{H}^{2}(\Pi) d k \rightarrow \int_{Q}^{\oplus} L_{2}(\mathbb{T}) d k$ on the LHS and the trace operator $H^{2}\left(\mathbb{R}^{3}\right) \rightarrow L_{2}(\mathbb{R})$ on the RHS. We denote the 'unperturbed' resolvent by $R_{0}(z, k):=\left(H_{0}(k)-z I\right)^{-1}$. In view of (2.7) we find

$$
\tilde{\mathcal{U}} \gamma R_{0}(z) \mathcal{U}^{*}=\int_{Q}^{\oplus} \gamma R_{0}(z, k) d k, \quad z \in \mathbb{C} \backslash[0, \infty) .
$$

Moreover, it turns out that

$$
\tilde{\mathcal{U}} T(z) \tilde{\mathcal{U}}^{*}=\int_{Q}^{\oplus} T(z, k) d k, \quad z \in \mathbb{C} \backslash[0, \infty) .
$$

Combining (3.4), (3.5) with (3.1) we conclude easily that

$$
T(\bar{z}, k)=T(z, k)^{*}, \quad T(z, k)-T(\zeta, k)=(\zeta-z)\left(\gamma R_{0}(\zeta, k)\right)\left(\gamma R_{0}(\bar{z}, k)\right)^{*}
$$

for all $k \in Q, z, \zeta \in \mathbb{C} \backslash\left[k^{2}, \infty\right)$. (Originally, these relations follow for $z \in$ $\mathbb{C} \backslash\left[k^{2}, \infty\right)$ and can be extended by analyticity to $z \in\left[0, k^{2}\right)$. Alternatively, they may be established directly.) It follows again from $\mathrm{Po}$ that there exists a self-adjoint operator $H(k)$ in $L_{2}(\Pi)$ such that its resolvent $R(z, k):=$ $(H(k)-z I)^{-1}$ satisfies

$$
\begin{aligned}
R(z, k)= & R_{0}(z, k)+\left(\gamma R_{0}(\bar{z}, k)\right)^{*}(T(z, k)+\sigma)^{-1} \gamma R_{0}(z, k), \\
& z \in \mathbb{C} \backslash\left[k^{2}, \infty\right), 0 \in \rho(T(z, k)+\sigma) .
\end{aligned}
$$


Combining this with (2.7), (3.4), (3.5) we obtain

$$
\mathcal{U} R(z) \mathcal{U}^{*}=\int_{Q}^{\oplus} R(z, k) d k, \quad z \in \rho(H),
$$

which implies (2.8). Finally, the characterization (2.6) is deduced from (2.3) as in EKo2].

\section{The Continuous spectrum of the operators $H(k)$}

4.1. Proof of Proposition 2.6. According to a result of Birman-Kreŭn (see $Y \mathrm{Ya}$ ) it suffices to prove that

$$
R\left(z_{0}, k\right)-R_{0}\left(z_{0}, k\right) \in \mathfrak{S}_{1},
$$

the trace class, for some $z_{0} \in \rho(H(k)) \cap \rho\left(H_{0}(k)\right)$. For $a>0$ sufficiently large the operator $T(-a, k)+\sigma$ is boundedly invertible. In view of (3.6) it suffices therefore to prove that

$$
\gamma R_{0}(-a, k) \in \mathfrak{S}_{2}
$$

the Hilbert-Schmidt class. For this recall that $R_{0}(z, k), z \in \mathbb{C} \backslash[0, \infty)$, is an integral operator with the kernel

$$
\begin{aligned}
r_{0}\left(x, y, x^{\prime}, y^{\prime}, z\right) & :=\frac{1}{(2 \pi)^{2}} \sum_{n \in \mathbb{Z}} e^{i n\left(x-x^{\prime}\right)} K_{0}\left(\sqrt{(n+k)^{2}-z}\left|y-y^{\prime}\right|\right), \\
(x, y),\left(x^{\prime}, y^{\prime}\right) & \in \Pi,
\end{aligned}
$$

where $K_{0}$ is the Macdonald function (or modified Bessel function of the second kind) of order zero (see $[\mathrm{AS}]$ ). Here and in the following we choose the branch of the square root on $\mathbb{C} \backslash(-\infty, 0]$ satisfying $\operatorname{Re} \sqrt{\cdot}>0$. It follows using Parseval's identity that

$$
\begin{aligned}
& \left\|\gamma R_{0}(-a, k)\right\|_{\mathfrak{S}_{2}}^{2}= \\
& \quad=\frac{1}{(2 \pi)^{4}} \int_{\mathbb{T}} \int_{\mathbb{T}} \int_{\mathbb{R}^{2}}\left|\sum_{n \in \mathbb{Z}} e^{i n\left(x-x^{\prime}\right)} K_{0}\left(\sqrt{(n+k)^{2}+a}|y|\right)\right|^{2} d y d x d x^{\prime}= \\
& \quad=\frac{1}{2 \pi} \sum_{n \in \mathbb{Z}} \frac{1}{(n+k)^{2}+a} \int_{0}^{\infty}\left|K_{0}(r)\right|^{2} r d r .
\end{aligned}
$$

Since the last integral is finite by properties of the Macdonald function, the proof of Proposition 2.6 is complete.

Remark 4.1. A more careful analysis shows that the singular values $s_{j}$ of the operator $R\left(z_{0}, k\right)-R_{0}\left(z_{0}, k\right)$ satisfy the estimate $\sup _{j} j^{1 / p} s_{j}<\infty$ with $p=\frac{1}{2}$. This should becompared with the exponent $p=\frac{2}{3}$ when the perturbation of $H_{0}$ is supported on a two-dimensional plane. (This can be seen as in the proof of Corollary 3.3 in [Fr1].) 
4.2. The limiting absorption principle for the unperturbed operator $H_{0}(k)$. In order to prove Proposition 2.7 we have to study the behaviour of the resolvent $R(z, k)$ as the spectral parameter approaches the real axis. The relation (3.6) suggests that we begin with the investigation of the unperturbed resolvent $R_{0}(z, k)$. Recall the definition of the threshold set $\tau(k)$ where the spectral multiplicity of the operator $H_{0}(k)$, and according to Proposition 2.6 also of $H(k)$, changes. Denote by $\Lambda$ the operator of multiplication by the function $\left(1+|y|^{2}\right)^{-1 / 2}$ in $L_{2}(\Pi)$.

Lemma 4.2. Let $k \in Q, \lambda \in \mathbb{R} \backslash \tau(k)$ and $s>\frac{1}{2}$. Then the operators

$$
\Lambda^{s} R_{0}(\lambda \pm i \epsilon, k) \Lambda^{s}, \quad \gamma R_{0}(\lambda \pm i \epsilon, k) \Lambda^{s}, \quad \epsilon>0,
$$

have limits in Hilbert-Schmidt norm as $\epsilon \rightarrow 0+$. The convergence is uniform in $\lambda$ from compact subsets of $\mathbb{R} \backslash \tau(k)$.

Proof. This follows in a straightforward way from the explicit expression (4.1) and standard properties of the Bessel function involved.

4.3. Proof of Proposition 2.7. Let $k \in Q$ be fixed. For $z \in \mathbb{C}_{+}$the operator $(T(z, k)-i)^{-1}$ exists, is compact and depends analytically on $z$. Moreover, for any $\lambda \in \mathbb{R} \backslash \tau(k)$ this family has an analytic extension to a neighbourhood of $\lambda$ in $\overline{\mathbb{C}_{-}}$. Applying the analytic Fredholm alternative (see Theorem VII.1.9 in $[\mathrm{K})$ to the family $(T(z, k)-i)^{-1}(\sigma+i)$ we conclude that there exists a set $\mathcal{N}_{+}(k)$, discrete in $\mathbb{R} \backslash \tau(k)$, such that the operators

$(T(\lambda+i \epsilon, k)+\sigma)^{-1}=\left(I+(T(\lambda+i \epsilon, k)-i)^{-1}(\sigma+i)\right)^{-1}(T(\lambda+i \epsilon, k)-i)^{-1}$ have a bounded limit as $\epsilon \rightarrow 0+$ for all $\lambda \in \mathbb{R} \backslash\left(\tau(k) \cup \mathcal{N}_{+}(k)\right)$. The limit is uniform for $\lambda$ from compact subsets of this set.

Combining this with relation (3.6) and Lemma 4.2 we see that the operators

$$
\Lambda^{s} R(\lambda+i \epsilon, k) \Lambda^{s}, \quad \epsilon>0,
$$

have limits as $\epsilon \rightarrow 0+$ for all $\lambda \in \mathbb{R} \backslash\left(\tau(k) \cup \mathcal{N}_{+}(k)\right.$ ) (in Hilbert-Schmidt norm). Moreover, the limit is uniform for $\lambda$ from compact subsets of this set. This implies (see [RS ) that $\sigma_{s c}(H(k)) \subset \overline{\tau(k) \cup \mathcal{N}_{+}(k)}$, and since the latter set is countable the assertion of Proposition 2.7 follows.

Remark 4.3. Denote by $T(\lambda+i 0, k), \lambda \in \mathbb{R} \backslash \tau(k)$, the boundary value of the operator function $T(z, k), z \in \mathbb{C}_{+}$. Then $\mathcal{N}_{+}(k)$ consists of the values $\lambda \in \mathbb{R} \backslash \tau(k)$ such that -1 is an eigenvalue of the operator $T(\lambda+i 0, k)^{-1} \sigma$. In the next subsection we will see that this is equivalent to $\lambda$ being a (nonthreshold) eigenvalue of $H(k)$.

\section{The Point SPeCtrum of the operators $H(k)$}

Since the perturbed operator in our case is not defined via a quadratic form we cannot use the usual Birman-Schwinger principle for the study of the eigenvalues of $H(k)$. In Subsection 5.1 we will prove a convenient substitute. 
5.1. Characterization of eigenvalues of $H(k)$. Let $k \in Q, \lambda \in \mathbb{R} \backslash \tau(k)$ and define

$$
\alpha_{n}(\lambda, k):=\frac{1}{4 \pi} \log \left|(n+k)^{2}-\lambda\right|-\varsigma, \quad n \in \mathbb{Z} .
$$

In the Hilbert space $L_{2}(\mathbb{T})$ we consider the operator

$$
\begin{gathered}
(A(\lambda, k) f)(x):=\frac{1}{\sqrt{2 \pi}} \sum_{n \in \mathbb{Z}} \alpha_{n}(\lambda, k) \hat{f}_{n} e^{i n x}+\sigma(x) f(x), \quad x \in \mathbb{T}, \\
\mathcal{D}(A(\lambda, k)):=\left\{f \in L_{2}(\mathbb{T}): \sum_{n \in \mathbb{Z}}\left(\log \left(1+n^{2}\right)\right)^{2}\left|\hat{f}_{n}\right|^{2}<\infty\right\} .
\end{gathered}
$$

In the case $\sigma \equiv 0$ we will denote this operator by $A_{0}(\lambda, k)$. Note that the operator in this case differs from the operator $T(\lambda+i 0, k)$ from Remark 4.3 only on the subspace $\left\{f \in L_{2}(\mathbb{T}): \hat{f}_{n}=0,(n+k)^{2}<\lambda\right\}$. The definition on that subspace is rather arbitrary (see Remark 5.2) and chosen only for technical convenience.

The compactness of the embedding of $\mathcal{D}(A(\lambda, k))$ in $L_{2}(\mathbb{T})$ implies that the operator $A(\lambda, k)$ has compact resolvent.

Now we characterize the non-threshold eigenvalues of the operator $H(k)$ as the values $\lambda$ for which 0 is an eigenvalue of the operator $A(\lambda, k)$. More precisely, we have

Proposition 5.1. Let $k \in Q$ and $\lambda \in \mathbb{R} \backslash \tau(k)$.

(1) Let $u \in \mathcal{N}(H(k)-\lambda I)$ and define

$$
u(x, y)=\frac{1}{\sqrt{2 \pi}} \sum_{(n+k)^{2}>\lambda} \hat{f}_{n} e^{i n x} K_{0}\left(\sqrt{(n+k)^{2}-\lambda}|y|\right), \quad(x, y) \in \Pi \backslash \Gamma .
$$

(2) Let $f \in \mathcal{N}(A(\lambda, k))$ such that $\hat{f}_{n}=0$ if $(n+k)^{2}<\lambda$ and define $u$ by (5.4).

Then $u \in \mathcal{N}(H(k)-\lambda I)$ and, moreover, (5.3) holds.

Remark 5.2. Note that the statement of Proposition 5.1 does not depend on the definition of $\alpha_{n}(\lambda, k)$ for $(n+k)^{2}<\lambda$. In particular, Remark 4.3 follows from Proposition [5.1]

Proof. Let $u \in \mathcal{N}(H(k)-\lambda I)$ and write

$$
u(x, y)=\frac{1}{\sqrt{2 \pi}} \sum_{n \in \mathbb{Z}} \hat{u}_{n}(y) e^{i n x}, \quad \hat{u}_{n}(y):=\frac{1}{\sqrt{2 \pi}} \int_{\mathbb{T}} u(x, y) e^{-i n x} d x .
$$

From $u \in \tilde{\Upsilon}$ it follows that $\hat{u}_{n} \in H_{l o c}^{2}\left(\mathbb{R}^{2} \backslash\{0\}\right) \cap L_{2}\left(\mathbb{R}^{2}\right)$ and that the limits

$$
\Xi \hat{u}_{n}:=-\lim _{y \rightarrow 0} \frac{1}{\log |y|} \hat{u}_{n}(y), \quad \Omega \hat{u}_{n}:=\lim _{y \rightarrow 0}\left(\hat{u}_{n}(y)+\log |y| \Xi \hat{u}_{n}\right),
$$


exist. Moreover, $\hat{u}_{n}$ satisfies

$$
-\Delta \hat{u}_{n}=\left(\lambda-(n+k)^{2}\right) \hat{u}_{n} \quad \text { in } \mathbb{R}^{2} \backslash\{0\} .
$$

It is well-known that this implies

$$
\hat{u}_{n}(y)= \begin{cases}0 & \text { if }(n+k)^{2}<\lambda, \\ c_{n} K_{0}\left(\sqrt{(n+k)^{2}-\lambda}|y|\right) & \text { if }(n+k)^{2}>\lambda .\end{cases}
$$

for some constants $c_{n} \in \mathbb{C}$. Now (see $[\mathrm{AS}$ )

$$
K_{0}\left(\sqrt{(n+k)^{2}-\lambda} \epsilon\right)+\log \epsilon \rightarrow-2 \pi \alpha_{n}(\lambda, k) \quad(\epsilon \rightarrow 0)
$$

implies that

$$
\begin{gathered}
f(x)=(\Xi u)(x)=\frac{1}{\sqrt{2 \pi}} \sum_{(n+k)^{2}>\lambda} c_{n} e^{i n x}, \\
(\Omega u)(x)=-\sqrt{2 \pi} \sum_{(n+k)^{2}>\lambda} \alpha_{n}(\lambda, k) c_{n} e^{i n x}=-2 \pi\left(A_{0}(\lambda, k) f\right)(x) .
\end{gathered}
$$

In particular, we have proven that $f \in \mathcal{D}\left(A_{0}(\lambda, k)\right)=\mathcal{D}(A(\lambda, k))$. Finally we conclude that $-2 \pi A(\lambda, k) f=\Omega u-2 \pi \sigma \Xi u=0$.

The proof of part (2) is easier and will be omitted.

We note that the operators $H(k)$ may have infinitely many (embedded) eigenvalues.

Example 5.3. Let $\sigma \equiv \alpha \in \mathbb{R}$ be constant. Then

$$
\sigma_{p}(H(k))=\left\{\xi(\alpha)+(n+k)^{2}: n \in \mathbb{Z}\right\} .
$$

This follows from Proposition 5.1 or directly by separation of variables using the results from $\mathrm{AGHH}$.

5.2. Proof of Proposition 2.8, Let $k$ be fixed throughout this subsection. First we will prove that the operator $H(k)$ has always as eigenvalue less or equal $\xi(\tilde{\sigma})+k^{2}$ where $\xi$ and $\tilde{\sigma}$ are given by (2.5), (2.9), respectively. Consider the normalized trial function $e_{0} \equiv \frac{1}{\sqrt{2 \pi}} \in L_{2}(\mathbb{T})$. Then

$$
\left(A(\lambda, k) e_{0}, e_{0}\right)=\alpha_{0}(\lambda, k)+\tilde{\sigma} .
$$

This is non-positive provided $\lambda \geq \xi(\tilde{\sigma})+k^{2}$.

On the other hand, the operator $A(\lambda, k)$ is positive definite provided $-\lambda$ is large. Since the eigenvalues of $A(\lambda, k)$ depend continuously on $\lambda$ there is a $\lambda_{0} \in\left(-\infty, k^{2}+\xi(\tilde{\sigma})\right]$ such that $A\left(\lambda_{0}, k\right)$ has eigenvalue 0. By Proposition 5.1 this proves the first part of Proposition 2.8.

To prove the second part we need the following

Lemma 5.4. Let $\sigma_{1} \in L_{\infty}(\mathbb{R})$ be real-valued and $2 \pi$-periodic and let $H_{1}$, $H_{1}(k)$ be the operators corresponding to $\sigma_{1}$. Then $\sigma_{1} \leq \sigma$ implies $H_{1} \leq H$, $H_{1}(k) \leq H(k)$. 
Proof. We consider only the case of the operators in $L_{2}\left(\mathbb{R}^{3}\right)$. It suffices to prove that for some $a>0$ one has $R(-a) \leq R_{1}(-a)$, where $R_{1}(z)=$ $\left(H_{1}-z I\right)^{-1}$. By the identity (3.2) and a similar identity for $R_{1}(-a)$ it suffices to prove $(T(-a)+\sigma)^{-1} \leq\left(T(-a)+\sigma_{1}\right)^{-1}$, which is immediate.

To complete the proof of Proposition 2.8 it suffices to take $\sigma_{1} \equiv \operatorname{ess-inf} \sigma$ and note (see Example 5.3) that the corresponding operator $H_{1}(k)$ has only a finite number of eigenvalues below $k^{2}$. By Lemma 5.4 and the variational principle the same holds true for the operator $H(k)$.

5.3. Complexification. In this subsection we fix $k \in Q, \lambda \in \mathbb{R} \backslash \tau(k)$ and assume in addition that $k \neq 0$. As in [FrSh] we choose $\delta \in(0,|k|)$ such that $(n+\kappa)^{2}-\lambda \neq 0$ for all $n \in \mathbb{Z}, \kappa \in[k-\delta, k+\delta]$, and note that there is a constant $C_{1}=C_{1}(k, \lambda, \delta)>0$ such that

$$
\left|(n+\mu)^{2}-\lambda\right| \geq C_{1}(1+|\operatorname{Im} \mu|)^{2}, \quad n \in \mathbb{Z}, \mu \in W,
$$

where we have put

$$
W:=\{\mu \in \mathbb{C}:|\operatorname{Re} \mu-k|<\delta\} .
$$

It follows that the functions $\alpha_{n}(\lambda,),. n \in \mathbb{Z}$, defined in (5.1) admit an analytic extension to $W$. This allows to define an m-sectorial operator $A(\lambda, \mu)$ for $\mu \in W$ by (5.2). We need the following result for $\mu$ with large imaginary part.

Lemma 5.5. Let $k, \lambda, \delta$ be as above. Then there exist constants $C_{2}=$ $C_{2}(k, \lambda, \delta), \eta_{0}>0$ such that for all $\mu \in W$ with $|\operatorname{Im} \mu| \geq \eta_{0}$ the operator $A(\lambda, \mu)$ is boundedly invertible and

$$
\left\|(A(\lambda, \mu))^{-1}\right\| \leq \frac{C_{2}}{\log (1+|\operatorname{Im} \mu|)} .
$$

Proof. From (5.5) we see that

$$
\begin{aligned}
\left|\alpha_{n}(\lambda, \mu)\right| & \geq \frac{1}{4 \pi} \log \left|(n+\mu)^{2}-\lambda\right|-\varsigma \geq \\
& \geq \frac{1}{2 \pi} \log (1+|\operatorname{Im} \mu|)-\left(\varsigma-\frac{1}{4 \pi} \log C_{1}\right),
\end{aligned}
$$

because $\varsigma>0$. We conclude that for large $|\operatorname{Im} \mu|$ the operator $A_{0}(\lambda, \mu)$ is boundedly invertible with

$$
\left\|\left(A_{0}(\lambda, \mu)\right)^{-1}\right\| \leq \frac{C_{3}}{\log (1+|\operatorname{Im} \mu|)},
$$

and we obtain the assertion by noting that

$$
(A(\lambda, \mu))^{-1}=\left(I+\left(A_{0}(\lambda, \mu)\right)^{-1} \sigma\right)^{-1}\left(A_{0}(\lambda, \mu)\right)^{-1}
$$

whenever $\left\|\left(A_{0}(\lambda, \mu)\right)^{-1} \sigma\right\|<1$.

Now we obtain easily the

Proof of Proposition [2.9. It suffices to repeat the arguments in the proof of Proposition 1.10 in [Fr2], replacing Proposition 3.5 there by our Lemma 5.5] 


\section{THE SECOND MODEL: AN INFINITE FAMILY OF LINES}

Now we would like to discuss a model of a "diffraction grating" consisting of periodically arranged wires. Our approach will be similar to the one outlined above and we emphasize these similarities by keeping the same notation for analogous objects. However, several constructions in the present case are technically more involved and we concentrate on these in the exposition.

6.1. Main result for the second model. It is convenient to denote now the coordinates in $\mathbb{R}^{3}$ by $(x, y), x \in \mathbb{R}^{2}, y \in \mathbb{R}$, and to put

$$
\Gamma:=\bigcup_{n \in \mathbb{Z}}\left\{\left(x_{1}, 2 \pi n, 0\right): x_{1} \in \mathbb{R}\right\} .
$$

As before let $\sigma$ be a real-valued, $2 \pi$-periodic function satisfying (2.1). We will construct a self-adjoint operator $H$ in $L_{2}\left(\mathbb{R}^{3}\right)$ corresponding to the formal expression $-\Delta+\sum_{n \in \mathbb{Z}} \sigma\left(x_{1}\right) \delta\left(x_{2}-2 \pi n\right) \delta(y)$, see the footnote in Subsection 2.1 Put $C_{\epsilon}:=\cup_{n \in \mathbb{Z}}\left\{(x, y) \in \mathbb{R}^{3}:\left(x_{2}+2 \pi n\right)^{2}+y^{2} \leq \epsilon^{2}\right\}$ for $\epsilon>0$. We denote by $\Upsilon$ the class of functions $u \in L_{2}\left(\mathbb{R}^{3}\right)$ satisfying

$$
u \in H^{2}\left(\mathbb{R}^{3} \backslash C_{\epsilon}\right) \quad \text { for all } \epsilon>0
$$

and such that for all $n \in \mathbb{Z}$ the limits

$$
\begin{aligned}
& \Xi_{n} u:=-\lim _{\left(x_{2}, y\right) \rightarrow 0} \frac{1}{\log \sqrt{x_{2}^{2}+y^{2}}} u\left(., x_{2}+2 \pi n, y\right), \\
& \Omega_{n} u:=\lim _{\left(x_{2}, y\right) \rightarrow 0}\left(u\left(., x_{2}+2 \pi n, y\right)+\log \sqrt{x_{2}^{2}+y^{2}} \Xi u\right)
\end{aligned}
$$

exist in the sense of distributions, belong to $L_{2}(\mathbb{R})$ and satisfy

$$
\sum_{n \in \mathbb{Z}}\left(\left\|\Xi_{n} u\right\|^{2}+\left\|\Omega_{n} u\right\|^{2}\right)<\infty
$$

We introduce the operators $\Xi, \Omega: L_{2}\left(\mathbb{R}^{3}\right) \rightarrow \sum_{n \in \mathbb{Z}}^{\oplus} L_{2}(\mathbb{R})$,

$$
\begin{aligned}
(\Xi u)_{n} & :=\Xi_{n} u, \quad(\Omega u)_{n}:=\Omega_{n} u, \\
\mathcal{D}(\Xi) & :=\mathcal{D}(\Omega):=\Upsilon .
\end{aligned}
$$

As before (see also Subsection 7.1 below) one constructs a self-adjoint operator $H$ in $L_{2}\left(\mathbb{R}^{3}\right)$ such that

$$
\begin{aligned}
H u & =-\Delta u \quad \text { in } \mathbb{R}^{3} \backslash \Gamma, \\
\mathcal{D}(H) & =\left\{u \in \Upsilon: \Delta u \in L_{2}\left(\mathbb{R}^{3} \backslash \Gamma\right), \Omega u-2 \pi \sigma \Xi u=0\right\} .
\end{aligned}
$$

We will again denote the standard self-adjoint realization of $-\Delta$ in $\mathbb{R}^{3}$ by $H_{0}$. Our main result is

Theorem 6.1. Theorems 2.1, 2.2, 2.3 hold also in the above situation. 
Remark 6.2. For simplicity we assume that our model is $2 \pi$-periodic with respect to both $x_{1}$ and $x_{2}$. Our argument extends easily to the case where the periods are different. The assumption (2.1) on $\sigma$ can be relaxed as before.

\subsection{Direct integral decomposition. Now we write}

$$
\Pi:=\left\{(x, y) \in \mathbb{R}^{3}: x \in[-\pi, \pi)^{2}\right\}
$$

and define $\tilde{H}^{2}(\Pi)$ and $\tilde{\Upsilon}$ in an obvious way. As before we consider $\Xi u, \Omega u$ for $u \in \tilde{\Upsilon}$ as functions in $L_{2}(\mathbb{T})$.

For any (two-dimensional) parameter $k \in Q:=\left[-\frac{1}{2}, \frac{1}{2}\right)^{2}$ there exists (see Subsection (7.3) a self-adjoint operator $H(k)$ in $L_{2}(\Pi)$ such that

$$
\begin{aligned}
H(k) u & =\left(\left(D_{x}+k\right)^{2}+D_{y}^{2}\right) u \quad \text { in } \Pi \backslash \Gamma, \\
\mathcal{D}(H(k)) & =\left\{u \in \tilde{\Upsilon}: \Delta u \in L_{2}(\Pi \backslash \Gamma), \Omega u-2 \pi \sigma \Xi u=0\right\} .
\end{aligned}
$$

Here $D_{x}=-i \nabla_{x}, D_{y}=-i \frac{\partial}{\partial y}$. Moreover, we denote by $H_{0}(k)$ the operator $\left(D_{x}+k\right)^{2}+D_{y}^{2}$ in $L_{2}(\Pi)$ with domain $\tilde{H}^{2}(\Pi)$.

The Gelfand transformation $\mathcal{U}: L_{2}\left(\mathbb{R}^{3}\right) \rightarrow \int_{Q}^{\oplus} L_{2}(\Pi) d k$ is in this case defined by

$$
(\mathcal{U} u)(k, x, y):=\sum_{n \in \mathbb{Z}^{2}} e^{-i\langle k, x+2 \pi n\rangle} u(x+2 \pi n, y), \quad k \in Q,(x, y) \in \Pi,
$$

and realizes the unitary equivalences

$$
\mathcal{U} H_{0} \mathcal{U}^{*}=\int_{Q}^{\oplus} H_{0}(k) d k, \quad \mathcal{U} H \mathcal{U}^{*}=\int_{Q}^{\oplus} H(k) d k
$$

As before the proof of Theorem 6.1 reduces to the following

Proposition 6.3. Propositions [2.6, 2.7, 2.8, 2.9 hold also in the above situation.

\section{Definition of the Operators in the SECOND MODEL}

7.1. Definition of the operator $H$. Let us start with some remarks concerning the definition of the operator $H$. We consider the trace operator $\gamma: H^{2}\left(\mathbb{R}^{3}\right) \rightarrow \sum_{n \in \mathbb{Z}}^{\oplus} L_{2}(\mathbb{R})$,

$$
(\gamma u)_{n}\left(x_{1}\right):=u\left(x_{1}, 2 \pi n, 0\right), \quad x_{1} \in \mathbb{R}, n \in \mathbb{Z} .
$$

The operator $T(z)$ will in this case be an operator in $\sum_{n \in \mathbb{Z}}^{\oplus} L_{2}(\mathbb{R})$. We need some preparations. For $z \in \mathbb{C} \backslash[0, \infty)$ we define pseudo-differential operators 
$T_{j}(z), j \in \mathbb{N}_{0}$, in $L_{2}(\mathbb{R})$ by

$$
\begin{aligned}
T_{0}(z) & :=\frac{1}{4 \pi} \log \left(D^{2}-z\right)-\varsigma I, \\
T_{j}(z) & :=-\frac{1}{2 \pi} K_{0}\left(2 \pi j\left(D^{2}-z\right)^{1 / 2}\right), \quad j \in \mathbb{N}, \\
\mathcal{D}\left(T_{0}(z)\right) & :=\left\{f \in L_{2}(\mathbb{R}): \int_{\mathbb{R}}\left(\log \left(1+\xi^{2}\right)\right)^{2}|\hat{f}(\xi)|^{2} d \xi<\infty\right\},
\end{aligned}
$$

Again we choose here and in all the following the principal branches of the logarithm and the square root on $\mathbb{C} \backslash(-\infty, 0]$.

Note that $T_{0}(z)$ coincides with the operator $T(z)$ from Subsection 3.1 . The following assertion shows in particular that the $T_{j}(z), j \in \mathbb{N}$, are bounded operators.

Lemma 7.1. Let $z \in \mathbb{C} \backslash[0, \infty)$. Then $\sum_{j \in \mathbb{N}}\left\|T_{j}(z)\right\|<\infty$.

Proof. For any $\epsilon>0$ there exists a $C_{\epsilon}>0$ such that

$$
\left|K_{0}(\zeta)\right| \leq C_{\epsilon} \frac{e^{-\operatorname{Re} \zeta}}{|\zeta|^{1 / 2}}, \quad|\zeta| \geq \epsilon
$$

(see [AS]). With $\epsilon:=2 \pi \inf _{\xi \in \mathbb{R}}\left|\xi^{2}-z\right|^{1 / 2}>0$ we find that for all $j \in \mathbb{N}$

$$
\left\|T_{j}(z)\right\|=\frac{1}{2 \pi} \sup _{\xi \in \mathbb{R}}\left|K_{0}\left(2 \pi j\left(\xi^{2}-z\right)^{1 / 2}\right)\right| \leq \tilde{C}_{\epsilon} \sup _{\xi \in \mathbb{R}} e^{-2 \pi j \operatorname{Re} \sqrt{\xi^{2}-z}} .
$$

Since $\operatorname{Re} \sqrt{\xi^{2}-z}$ is bounded away from 0 the assertion follows.

¿From Lemma 7.1 and Schur's lemma one finds that the operator $T(z)$ in $\sum_{n \in \mathbb{Z}}^{\oplus} L_{2}(\mathbb{R})$,

$$
\begin{aligned}
(T(z) f)_{n} & :=\sum_{m \in \mathbb{Z}} T_{|n-m|}(z) f_{m}, \quad n \in \mathbb{Z}, \\
\mathcal{D}(T(z)) & :=\left\{f \in \sum_{n \in \mathbb{Z}}^{\oplus} L_{2}(\mathbb{R}): \sum_{n \in \mathbb{Z}} \int_{\mathbb{R}}\left(\log \left(1+\xi^{2}\right)\right)^{2}\left|\hat{f}_{n}(\xi)\right|^{2} d \xi<\infty\right\},
\end{aligned}
$$

is well-defined. Moreover, one verifies that for $z, \zeta \in \mathbb{C} \backslash[0, \infty)$

$$
T(\bar{z})=T(z)^{*}, \quad T(z)-T(\zeta)=(\zeta-z)\left(\gamma R_{0}(\zeta)\right)\left(\gamma R_{0}(\bar{z})\right)^{*} .
$$

Again by $[\mathrm{Po}$ this implies that there exists a self-adjoint operator $H$ in $L_{2}\left(\mathbb{R}^{3}\right)$ such that its resolvent $R(z):=(H-z I)^{-1}$ is related to $R_{0}(z)$ by

$$
\begin{aligned}
R(z)= & R_{0}(z)+\left(\gamma R_{0}(\bar{z})\right)^{*}(T(z)+\sigma)^{-1} \gamma R_{0}(z), \\
& z \in \mathbb{C} \backslash[0, \infty), 0 \in \rho(T(z)+\sigma) .
\end{aligned}
$$

Here we understand $\sigma$ as an operator in $\sum_{n \in \mathbb{Z}}^{\oplus} L_{2}(\mathbb{R})$ acting according to $(\sigma f)_{n}=\sigma f_{n}$ for $f=\left(f_{n}\right) \in \sum_{n \in \mathbb{Z}}^{\oplus} L_{2}(\mathbb{R})$.

By the proof of Lemma 7.1 one easily finds that $T(-a)+\sigma$ is positive definite 
for all sufficiently large $a$, and hence $H$ is lower semibounded.

Finally, one shows that $H$ satisfies (6.2). This follows from the identities

$$
\Xi\left(\gamma R_{0}(\bar{z})\right)^{*}=\frac{1}{2 \pi} I, \quad \Omega\left(\gamma R_{0}(\bar{z})\right)^{*}=-T(z) .
$$

7.2. Auxiliary material. Before we can explain the construction of the operators $H(k)$ we need to collect some material on point interactions in a two-dimensional strip. Note that our approach is somewhat different from the one adopted in Section III.4 in AGHH. Having in mind the later application we denote the coordinates in $\mathbb{R}^{2}$ by $\left(x_{2}, y\right)$, the quasi-momentum by $k_{2} \in Q^{\prime}:=\left[-\frac{1}{2}, \frac{1}{2}\right)$ and put

$$
\Pi^{\prime}:=[-\pi, \pi) \times \mathbb{R} .
$$

For $z \in \mathbb{C} \backslash\left[k_{2}^{2}, \infty\right)$ we introduce the function

$$
\begin{aligned}
\psi\left(x_{2}, y, z, k_{2}\right):= & \frac{1}{2} \sum_{n \in \mathbb{Z}} \frac{e^{i n x_{2}}}{\sqrt{\left(n+k_{2}\right)^{2}-z}} e^{-\sqrt{\left(n+k_{2}\right)^{2}-z}|y|}, \\
& \left(x_{2}, y\right) \in \Pi^{\prime} \backslash\{(0,0)\} .
\end{aligned}
$$

This function belongs to $L_{2}\left(\Pi^{\prime}\right)$ and is smooth away from $(0,0)$. Moreover,

Lemma 7.2. Let $k_{2} \in Q^{\prime}, z \in \mathbb{C}$ and assume that $u \in L_{2}\left(\Pi^{\prime}\right)$ is a periodic (with respect to $x_{2}$ ) solution of

$$
\left(\left(D_{x_{2}}+k_{2}\right)^{2}+D_{y}^{2}\right) u=z u \quad \text { in } \Pi^{\prime} \backslash\{(0,0)\} .
$$

If $z \in\left[k^{2}, \infty\right)$ then $u \equiv 0$, and if $z \in \mathbb{C} \backslash\left[k_{2}^{2}, \infty\right)$ then

$$
u=c \psi\left(\cdot, z, k_{2}\right), \quad c \in \mathbb{C} .
$$

By a periodic (with respect to $x_{2}$ ) solution of (7.3) we mean that the test functions in the distributional definition of a solution are not required to vanish near $\partial \Pi^{\prime}$ but only to be periodic (with respect to $x_{2}$ ).

The proof of Lemma 7.2 follows easily by Fourier transformation and the fact that $\left(\left(D_{x_{2}}+k_{2}\right)^{2}+D_{y}^{2}-z\right) u$ is a distribution supported on $\{(0,0)\}$ and hence coincides with a finite linear combination of derivates of the $\delta$ distribution.

For $z \in \mathbb{C} \backslash[0, \infty)$ the following alternative expression for $\psi(\cdot, z, k)$ exists,

$$
\psi\left(x_{2}, y, z, k_{2}\right)=\sum_{m \in \mathbb{Z}} e^{-i k_{2}\left(x_{2}+2 \pi m\right)} K_{0}\left(\sqrt{-z} \sqrt{\left(x_{2}+2 \pi m\right)^{2}+y^{2}}\right) .
$$

Indeed, this follows from

$$
\frac{1}{\pi} \int K_{0}\left(\sqrt{-z} \sqrt{x_{2}^{2}+y^{2}}\right) e^{-i \xi x_{2}} d x_{2}=\frac{e^{-\sqrt{\xi^{2}-z}|y|}}{\sqrt{\xi^{2}-z}}, \quad \xi \in \mathbb{R},
$$

by the Poisson summation formula.

Put $C_{\epsilon}^{\prime}:=\left\{\left(x_{2}, y\right) \in \mathbb{R}^{2}: x_{2}^{2}+y^{2} \leq \epsilon^{2}\right\}$. We denote by $\tilde{\Upsilon}^{\prime}$ the class of functions $u \in L_{2}\left(\Pi^{\prime}\right)$ satisfying

$$
u \in \tilde{H}^{2}\left(\Pi^{\prime} \backslash C_{\epsilon}^{\prime}\right) \quad \text { for all } \epsilon>0
$$


and such that the limits

$$
\begin{aligned}
& \Xi u:=-\lim _{\left(x_{2}, y\right) \rightarrow 0} \frac{1}{\log \sqrt{x_{2}^{2}+y^{2}}} u\left(x_{2}, y\right), \\
& \Omega u:=\lim _{\left(x_{2}, y\right) \rightarrow 0}\left(u\left(x_{2}, y\right)+\log \sqrt{x_{2}^{2}+y^{2}} \Xi u\right)
\end{aligned}
$$

exist. It is not difficult to verify that $\psi\left(\cdot, z, k_{2}\right) \in \tilde{\Upsilon}^{\prime}$ with $\Xi \psi(\cdot, z, k)=1$ and where

$$
t\left(z, k_{2}\right):=-\frac{1}{2 \pi} \Omega \psi\left(\cdot, z, k_{2}\right), \quad z \in \mathbb{C} \backslash\left[k_{2}^{2}, \infty\right),
$$

satisfies the relation

$$
t\left(z, k_{2}\right)=t(-1,0)-\frac{1}{2} \sum_{n \in \mathbb{Z}}\left(\frac{1}{\sqrt{\left(n+k_{2}\right)^{2}-z}}-\frac{1}{\sqrt{n^{2}+1}}\right) .
$$

Moreover, from (7.4) and the properties of $K_{0}$ (see AS) one deduces that for $z \in \mathbb{C} \backslash[0, \infty)$

$$
t\left(z, k_{2}\right)=\frac{1}{4 \pi} \log (-z)-\varsigma-\frac{1}{\pi} \sum_{j \in \mathbb{N}} \cos \left(2 \pi j k_{2}\right) K_{0}(2 \pi j \sqrt{-z}) .
$$

We close this subsection with an estimate that will be useful in the proof of absolute continuity. By the same arguments as in the proof of Lemma 7.1 we deduce from (17.6) the following

Lemma 7.3. For any $\epsilon>0$ there is a constant $C=C(\epsilon)>0$ such that for all $z \in \mathbb{C} \backslash[0, \infty)$ with $\operatorname{Re} \sqrt{-z} \geq \epsilon$ and all $k_{2} \in \overline{Q^{\prime}}$ one has

$$
\left|t\left(z, k_{2}\right)-\frac{1}{4 \pi} \log (-z)\right| \leq C .
$$

Remark 7.4. Note that the subtraction of the terms $\left(n^{2}+1\right)^{-1 / 2}$ in (7.5) is a renormalization of the divergent sum $\sum\left(\left(n+k_{2}\right)^{2}-z\right)^{-1 / 2}$. A different, but equivalent renormalization is chosen in Theorem III.4.8 in [AGHH].

7.3. Definition of the operators $H(k)$. Again we denote by $\gamma$ the trace operator $\tilde{H}^{2}(\Pi) \rightarrow L_{2}(\mathbb{T})$. For $k \in Q, z \in \mathbb{C} \backslash\left[|k|^{2}, \infty\right)$ we consider the pseudo-differential operator $T(z, k)$ in $L_{2}(\mathbb{T})$,

$$
\begin{aligned}
T(z, k) & :=t\left(z-\left(D_{x_{1}}+k_{1}\right)^{2}, k_{2}\right), \\
\mathcal{D}(T(z, k)) & :=\left\{f \in L_{2}(\mathbb{T}): \sum_{n \in \mathbb{Z}}\left(\log \left(1+n^{2}\right)\right)^{2}\left|\hat{f}_{n}\right|^{2}<\infty\right\} .
\end{aligned}
$$

It follows from Lemma 7.3 that $T(z, k)$ is closed and lower semibounded and has compact resolvent. Our next goal is to show that these operators appear as fibers in the direct integral decomposition of the operator $T(z)$. For this 
purpose consider the unitary operator $\tilde{\mathcal{U}}: \sum_{n \in \mathbb{Z}}^{\oplus} L_{2}(\mathbb{R}) \rightarrow \int_{Q}^{\oplus} L_{2}(\mathbb{T}) d k$, defined for smooth $f$ by

$$
(\tilde{\mathcal{U}} f)\left(k, x_{1}\right):=\sum_{m \in \mathbb{Z}^{2}} e^{-i k_{1} x_{1}-2 \pi i\langle k, m\rangle} f_{m_{2}}\left(x_{1}+2 \pi m_{1}\right), \quad k \in Q, x_{1} \in \mathbb{T} .
$$

We note that on $H^{2}\left(\mathbb{R}^{3}\right)$ one has the identity

$$
\gamma \mathcal{U}=\tilde{\mathcal{U}} \gamma
$$

with an obvious meaning of the trace operator $\gamma$ on the different sides of the equality. Moreover, it turns out that

$$
\tilde{\mathcal{U}} T(z) \tilde{\mathcal{U}}^{*}=\int_{Q}^{\oplus} T(z, k) d k, \quad z \in \mathbb{C} \backslash[0, \infty) .
$$

Similarly as in Subsection 3.2 we deduce from relations (17.7), (77.8) that there exists a self-adjoint operator $H(k)$ in $L_{2}(\Pi)$ such that its resolvent $R(z, k):=(H(k)-z I)^{-1}$ is related to $R_{0}(z, k):=\left(H_{0}(k)-z I\right)^{-1}$ by

$$
\begin{aligned}
R(z, k)= & R_{0}(z, k)+\left(\gamma R_{0}(\bar{z}, k)\right)^{*}(T(z, k)+\sigma)^{-1} \gamma R_{0}(z, k), \\
& z \in \mathbb{C} \backslash\left[k^{2}, \infty\right), 0 \in \rho(T(z, k)+\sigma),
\end{aligned}
$$

and that this operator satisfies (6.3) and (6.4).

8. The SPeCtrum of The OPERATORS $H(k)$ IN THE SECOND MOdeL

8.1. The continuous spectrum of $H(k)$. The analogue of Proposition 2.6] follows exactly as before using the explicit form of the 'unperturbed' resolvent kernel

$$
\begin{aligned}
r_{0}\left(x, y, x^{\prime}, y^{\prime}, z, k\right)= & \frac{1}{8 \pi^{2}} \sum_{n \in \mathbb{Z}^{2}} e^{i\left\langle n, x-x^{\prime}\right\rangle} \frac{e^{-\sqrt{|n+k|^{2}-z}\left|y-y^{\prime}\right|}}{\sqrt{|n+k|^{2}-z}}, \\
& (x, y),\left(x^{\prime}, y^{\prime}\right) \in \Pi .
\end{aligned}
$$

We note that the assertion of Remark 4.1 remains true.

To establish the analogue of Proposition 2.7 we proceed again as before taking into account that for any $m \in \mathbb{Z}, k \in Q, \lambda \in \mathbb{R} \backslash \tau(k)$, where now

$$
\tau(k):=\left\{|n+k|^{2}: n \in \mathbb{Z}^{2}\right\},
$$

the function $t\left(\cdot-\left(m+k_{1}\right)^{2}, k_{2}\right)$ has an analytic extension from $\mathbb{C}_{+}$to a neighbourhood of $\lambda$ in $\overline{\mathbb{C}_{-}}$. This is easily seen from (7.5).

8.2. Characterization of eigenvalues of $H(k)$. We turn to the point spectrum of the operators $H(k)$ and derive an analogue of Proposition 5.1 . For $k \in Q, \lambda \in \mathbb{R} \backslash \tau(k)$ we define

$$
\alpha_{n}(\lambda, k):=t\left(-\left|\left(n+k_{1}\right)^{2}+k_{2}^{2}-\lambda\right|+k_{2}^{2}, k_{2}\right), \quad n \in \mathbb{Z} .
$$


In the Hilbert space $L_{2}(\mathbb{T})$ we consider the operator

$$
\begin{aligned}
(A(\lambda, k) f)(x) & :=\frac{1}{\sqrt{2 \pi}} \sum_{n \in \mathbb{Z}} \alpha_{n}(\lambda, k) \hat{f}_{n} e^{i n x}+\sigma(x) f(x), \quad x \in \mathbb{T}, \\
\mathcal{D}(A(\lambda, k)) & :=\left\{f \in L_{2}(\mathbb{T}): \sum_{n \in \mathbb{Z}}\left(\log \left(1+n^{2}\right)\right)^{2}\left|\hat{f}_{n}\right|^{2}<\infty\right\} .
\end{aligned}
$$

As for the operators $T(z, k)$ one checks that $A(\lambda, k)$ is closed and lower semibounded and has compact resolvent. Our main tool in the investigation of the point spectrum of $H(k)$ will be

Proposition 8.1. Let $k \in Q$ and $\lambda \in \mathbb{R} \backslash \tau(k)$.

(1) Let $u \in \mathcal{N}(H(k)-\lambda I)$ and define

$$
u(x, y)=\frac{1}{\sqrt{2 \pi}} \sum_{\left(n+k_{1}\right)^{2}>\lambda-k_{2}^{2}} \hat{f}_{n} e^{i n x_{1}} \psi\left(x_{2}, y,\left(n+k_{1}\right)^{2}-\lambda, k_{2}\right),(x, y) \in \Pi \backslash \Gamma .
$$

(2) Let $f \in \mathcal{N}(A(\lambda, k))$ such that $\hat{f}_{n}=0$ if $\left(n+k_{1}\right)^{2}<\lambda-k_{2}^{2}$ and define $u$ by (8.4).

Then $u \in \mathcal{N}(H(k)-\lambda I)$ and, moreover, (8.3) holds.

Proceeding as in the proof of Proposition 5.1 this follows easily by Fourier transformation from Lemma 7.2

Again the operators $H(k)$ may have infinitely many (embedded) eigenvalues.

Example 8.2. Let $\sigma \equiv \alpha \in \mathbb{R}$ be constant. It follows from (7.5) and Lemma 7.3 that the function $t\left(\cdot, k_{2}\right)$ is continuous and decreasing on $\left(-\infty, k_{2}^{2}\right)$ with

$$
\lim _{\lambda \rightarrow k_{2}^{2}-} t\left(\lambda, k_{2}\right)=-\infty, \quad \lim _{\lambda \rightarrow-\infty} t\left(\lambda, k_{2}\right)=\infty .
$$

Hence there exists a unique $\lambda\left(\alpha, k_{2}\right) \in\left(-\infty, k_{2}^{2}\right)$ such that

$$
t\left(\lambda\left(\alpha, k_{2}\right), k_{2}\right)+\alpha=0
$$

¿From Proposition 8.1 it follows that

$$
\sigma_{p}(H(k))=\left\{\lambda\left(\alpha, k_{2}\right)+\left(n+k_{1}\right)^{2}: n \in \mathbb{Z}\right\} .
$$

This result (in equivalent notation) may also be deduced from the twodimensional result in $\mathrm{AGHH}$ by separation of variables.

For the proof of the analogue of Proposition 2.8 we proceed exactly as before. Using the trial function $e_{0}$ we find that $H(k)$ has an eigenvalue less or equal $\lambda\left(\tilde{\sigma}, k_{2}\right)+k_{1}^{2}$, where $\tilde{\sigma}$ is given by (2.9) and $\lambda\left(\cdot, k_{2}\right)$ was constructed in Example 8.2. Since $H_{1}(k)$, the operator corresponding to $\sigma_{1} \equiv \operatorname{ess-inf} \sigma$, has only finitely many eigenvalues an analogue of Lemma 5.4 finishes the proof of the analogue of Proposition 2.8. 
Finally, we turn to the proof of the analogue of Proposition 2.9 Again we will construct an analytic extension of the operators $A\left(\lambda, k_{1}, k_{2}\right)$ with respect to the variable $k_{1}$. The new ingredient here is that we replace the 'complicated' symbol $\alpha_{n}(\lambda, k)$ by the explicit $(4 \pi)^{-1} \log \left(\left(n+k_{1}\right)^{2}-\lambda\right)$ (which already appeared in the first part of our analysis). This requires careful estimates which are uniform in $n$ and in the (complex) parameter $k_{1}$.

Now we describe the details of our construction. Similarly as in Subsection [5.3] we fix $k \in Q, \lambda \in \mathbb{R} \backslash \tau(k)$ and assume that $k_{1} \neq 0$. We choose $\delta \in\left(0,\left|k_{1}\right|\right)$ satisfying $(n+\mu)^{2}+k_{2}^{2}-\lambda \neq 0$ for all $n \in \mathbb{Z}, \kappa \in\left[k_{1}-\delta, k_{1}+\delta\right]$, and put

$$
W:=\left\{\mu \in \mathbb{C}:\left|\operatorname{Re} \mu-k_{1}\right|<\delta\right\} .
$$

For $n \in \mathbb{Z}$ we consider the functions

$$
s_{n}(\mu):= \begin{cases}-(n+\mu)^{2}+\lambda, & \text { if }\left(n+k_{1}\right)^{2}+k_{2}^{2}-\lambda>0, \\ (n+\mu)^{2}-\lambda+2 k_{2}^{2}, & \text { if }\left(n+k_{1}\right)^{2}+k_{2}^{2}-\lambda<0 .\end{cases}
$$

We need the technical

Lemma 8.3. There are constants $\eta_{0}, \epsilon>0$ such that for all $n \in \mathbb{Z}, \mu \in W$ with $|\operatorname{Im} \mu| \geq \eta_{0}$ one has $s_{n}(\mu) \in \mathbb{C} \backslash[0, \infty)$ and

$$
\operatorname{Re} \sqrt{-s_{n}(\mu)} \geq \epsilon(1+|n|), \quad n \in \mathbb{Z}, \mu \in W,|\operatorname{Im} \mu| \geq \eta_{0} .
$$

Proof. We will write $\mu=\kappa+i \eta$ with $\kappa, \eta \in \mathbb{R}$. Introduce

$$
J:=\left\{n \in \mathbb{Z}:(n+\kappa)^{2}>\lambda \quad \forall\left|\kappa-k_{1}\right| \leq \delta\right\} .
$$

The set $\mathbb{Z} \backslash J$ is finite and for $n$ from that set the assertion is readily verified. Hence we will now consider only $n \in J$. In particular, we have $s_{n}(\mu)=$ $-(n+\mu)^{2}+\lambda$. Moreover, we will use the elementary estimate

$$
\left|(n+\mu)^{2}-\lambda\right| \geq c_{1} \min \left\{(1+|n|)^{2},(1+|\eta|)^{2}\right\}, \quad n \in J, \mu \in W .
$$

First, we assume that

$$
\operatorname{Re} s_{n}(\mu)=\eta^{2}-(n+\kappa)^{2}+\lambda \leq 0,
$$

and consequently

$$
\operatorname{Re} \sqrt{-s_{n}(\mu)}=\sqrt{\left|(n+\mu)^{2}-\lambda\right|} \cos \left(\frac{1}{2} \arctan \frac{2 \eta(n+\kappa)}{(n+\kappa)^{2}-\eta^{2}-\lambda}\right)
$$

(where $\left.\arctan ( \pm \infty)=\mp \frac{\pi}{2}\right)$. Noting that the cosine factor is bounded away from 0 the assertion follows immediately from (8.5).

Now we assume that the opposite inequality in (8.6) holds and consequently

$$
\operatorname{Re} \sqrt{-s_{n}(\mu)}=\sqrt{\left|(n+\mu)^{2}-\lambda\right|} \sin \left(\frac{1}{2} \arctan \frac{2|\eta(n+\kappa)|}{\eta^{2}-(n+\kappa)^{2}+\lambda}\right) .
$$

If, say, $2|\eta(n+\kappa)| \geq \eta^{2}-(n+\kappa)^{2}+\lambda$ then the sine factor is bounded away from 0 and the assertion follows again by (8.5). Otherwise if $2|\eta(n+\kappa)|$ 
$<\eta^{2}-(n+\kappa)^{2}+\lambda$ we note that there is a constant $c_{2}>0$ such that $\sin \left(\frac{1}{2} \arctan x\right) \geq c_{2} x$ for all $0 \leq x \leq 1$. Hence

$$
\operatorname{Re} \sqrt{-s_{n}(\mu)} \geq 2 c_{2} \sqrt{\left|(n+\mu)^{2}-\lambda\right|} \frac{|\eta(n+\kappa)|}{\eta^{2}-(n+\kappa)^{2}+\lambda} .
$$

Using now (8.5) and the estimate

$$
\frac{|\eta(n+\kappa)|}{\eta^{2}-(n+\kappa)^{2}+\lambda} \geq \frac{|n+\kappa|}{|\eta|} \geq c_{3} \frac{1+|n|}{|\eta|}
$$

we obtain the assertion.

Since the $s_{n}$ assume values in $\mathbb{C} \backslash\left[k_{2}^{2}, \infty\right)$, it follows from our discussion in Subsection 7.2 that $\alpha_{n}\left(\lambda, \mu, k_{2}\right):=t_{n}\left(s_{n}(\mu), k_{2}\right)$ defines an analytic function of $\mu \in W$. This allows to define an m-sectorial operator $A(\lambda, \mu)$ for $\mu \in W$ by (8.2). The analogue of Proposition 2.9 will follow as before if we can establish

Lemma 8.4. Lemma 5.5 holds also in the above situation.

Proof. By Lemmas 7.3 and 8.3 we have

$$
\left|\alpha_{n}\left(\lambda, \mu, k_{2}\right)-\frac{1}{4 \pi} \log \left(-s_{n}(\mu)\right)\right| \leq C, \quad n \in \mathbb{Z}, \mu \in W,|\operatorname{Im} \mu| \geq \eta_{0} .
$$

Using this one can proceed as in the proof of Lemma 5.5.

Remark 8.5. Assume $\lambda<k_{1}^{2}$ for simplicity. A closer look at the proof of Lemma 7.3 yields that we have

$$
\left|\alpha_{n}\left(\lambda, k_{1}, k_{2}\right)-\frac{1}{4 \pi} \log \left(\left(n+k_{1}\right)^{2}-\lambda\right)+\varsigma\right| \leq C e^{-c|n|}, \quad n \in \mathbb{Z},
$$

(even uniformly in $k_{1} \in W$ ). This reflects the physical fact that the interaction of the wires, being of a tunneling nature, decreases exponentially fast with their distance $2 \pi|n|$.

Acknowledgments. The research was supported in part by ASCR and its Grant Agency within the projects IRP AV0Z10480505 and A100480501. The second author acknowledges gratefully a partial support through the ESF SPECT programme.

\section{REFERENCES}

[AS] M.S. Abramowitz, I.A. Stegun, Handbook of mathematical functions with formulas, graphs, and mathematical tables. Reprint of the 1972 edition, Dover, New York, 1992.

[AGHH] S. Albeverio, F. Gesztesy, R. Høegh-Krohn, H. Holden, Solvable models in quantum mechanics. Second edition, with an appendix by P. Exner, AMS Chelsea Publishing, volume 350, American Mathematical Society, Providence, 2005.

[BeDuE] F. Bentosela, P. Duclos, P. Exner, Absolute continuity in periodic thin tubes and strongly coupled leaky wires, Lett. Math. Phys. 65 (2003), 75-82.

[EKo1] P. Exner, S. Kondej, Curvature-induced bound states for a $\delta$ interaction supported by a curve in $\mathbb{R}^{3}$, Ann. H. Poincaré 3 (2002), 967-981. 
[EKo2] P. Exner, S. Kondej, Strong-coupling asymptotic expansion for Schroedinger operators with a singular interaction supported by a curve in $\mathbb{R}^{3}$, Rev. Math. Phys 16 (2004), 559-582.

[FiKl] N. Filonov, F. Klopp, Absolute continuity of the spectrum of a Schrödinger operator with a potential which is periodic in some directions and decays in others, Doc. Math. 9 (2004), 107-121; Erratum: ibd., 135-136.

[Fr1] R. L. Frank, On the scattering theory of the Laplacian with a periodic boundary condition. I. Existence of wave operators, Doc. Math. 8 (2003), 547-565.

[Fr2] R. L. Frank, On the Laplacian in the halfspace with a periodic boundary condition, Ark. Mat., to appear.

[FrSh] R. L. Frank, R. G. Shterenberg, On the scattering theory of the Laplacian with a periodic boundary condition. II. Additional channels of scattering, Doc. Math. 9 (2004), 57-77.

[K] T. Kato, Perturbation theory for linear operators. Reprint of the 1980 edition, Springer, Berlin, 1995.

[Po] A. Posilicano, A Krein-like formula for singular perturbations of self-adjoint operators and applications, J. Funct. Anal. 183 (2001), 109-147.

[RS] M. Reed, B. Simon, Methods of modern mathematical phsics. IV. Analysis of operators, Academic Press, New York, 1978.

[T] L. Thomas, Time dependent approach to scattering from impurities in a crystal, Comm. Math. Phys. 33 (1973), 335-343.

[Ya] D. R. Yafaev, Mathematical scattering theory, American Mathematical Society, Providence, 1992.

Department of Theoretical Physics, Nuclear Physics Institute, Academy of Sciences, 25058 Rez near Prague, Czech Republic

E-mail address: exner@ujf.cas.cz

Royal Institute of Technology, Department of Mathematics, LindstedtsVÄGen 25, 10044 Stockholm, Sweden

E-mail address: rupert@math.kth.se 